\title{
Surface Acoustic Wave Resonators as Passive Buried Sensors
}

\author{
J.-M Friedt \\ and T. Rétornaz \\ Senseor, Besançon, France \\ Email: jmfriedt@femto-st.fr
}

\author{
G. Martin, T. Laroche, \\ É. Carry, and S. Ballandras \\ FEMTO-ST, Besançon, France \\ Email: ballandrefemto-st.fr
}

\author{
J.-P. Simonnet \\ Laboratoire de \\ ChronoEnvironnement, \\ Franche Comté University, \\ Besançon, France
}

\begin{abstract}
Acoustic wave devices are well known passive transducers for probing through a wireless link a physical quantity. Amongst the two main classes of designs - resonators and delay lines - the former have the advantage of providing informations in a narrow band signal and are hence compatible with an interrogation strategy compliant with radiofrequency (RF) emission regulations, while the latter are probed by a short RF pulse with larger instantaneous energy and shorter response time. We here demonstrate the measurement of temperature using the two configurations, and more specifically for sensors buried in soil. While we demonstrate long term stability and ruggedness of packaged resonators, and signal to noise ratio compatible with the envisioned application, the interrogation range in insufficient for most purposes and we focus towards the use of delay lines. Indeed, the interrogation method of the latter is similar to that used by ground penetrating RADAR (GPR) which displays interrogation ranges in the meter to tens of meters in the lower RF range, depending on soil water content, permittivity and conductivity.
\end{abstract}

\section{INTRODUCTION}

Within the framework of wireless sensors, surface acoustic wave piezoelectric devices provide unique performances in terms of roughness and autonomy with respect to active devices (better temperature stability compared to CMOS devices, lack of battery), and better interrogation range than RFID passive tags. The use of piezoelectric delay lines and resonators for monitoring physical quantities such as temperature, strain, torque and pressure have already been demonstrated. The short interrogation time (microsecond to millisecond range) is compatible with the use of quickly moving sensors, but ofter the size and metallic environment of the radiofrequency antenna are challenges to the interrogation range. Here we are interested in using surface acoustic wave (SAW) resonators and delay lines as buried sensors: while interrogation speed is hardly an issue, the interrogation range with define the performances of the sensors and the range of use. If only a few centimeters are reached, application are limited to concrete surface properties monitoring, road aging or near surface soil properties. The application range is greatly widened if interrogation ranges in the tens of meters can be reached [1], since deep soil properties are then reachable. We base our analysis of the use of SAW device as buried sensor on our own experiments and literature concerning Ground Penetrating Radar (GPR). The latter technique is widely used for monitoring dielectric interfaces in buried structures, with range dependant upon the probe electromagnetic pulse duration and dielectric properties of the soil. We focus on providing complementary informations from sensors with interrogation techniques compatible with GPR, following a strategy commonly known as cooperative target [2].

\section{BURIED RESONATORS AS PASSIVE TEMPERATURE SENSORS}

Three dual 433-MHz surface acoustic wave resonators (one reference frequency and one measurement frequency within the $1.7-\mathrm{MHz}$ wide ISM band) packaged in $5 \times 5 \mathrm{~mm}^{2}$ ceramic packages were buried in clay after being connected to dipole antenna whose length was adjusted prior to installation in soil assuming a relative permittivity of 10 . The purpose of this experiment was to validate the survival of sensors buried in soil and the evolution of the radiofrequency link quality over time, as a function of temperature or climatic conditions (i.e. moisture level in soil).

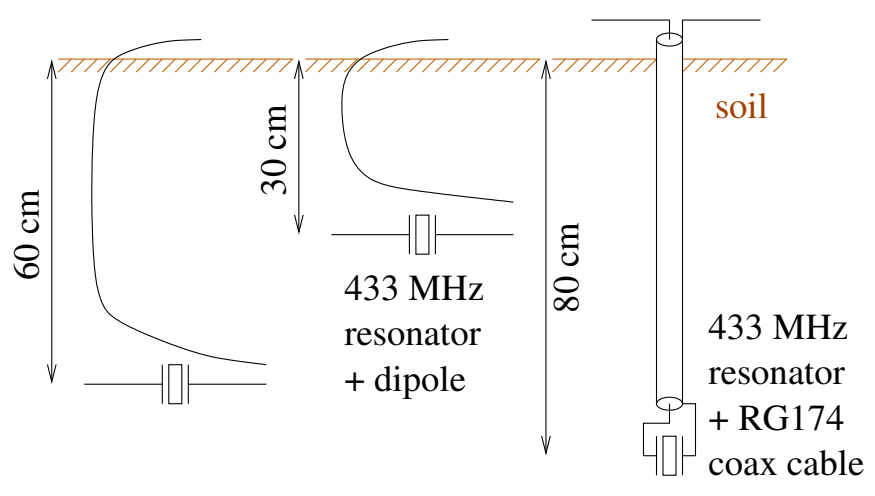

Fig. 1. Experimental configuration: the $30 \mathrm{~cm}$ and $60 \mathrm{~cm}$ deep devices are SAW resonators soldered to a $2 \times 5$ long dipole, buried in clay with a conducting wire located in the hole but neither electrically connected to the sensor nor to the interrogation unit. The $80 \mathrm{~cm}$ deep resonator was soldered to an RG174 coaxial cable protruding from ground as an open-feed.

The first observation during installation of the experiment is that an interrogation unit generating $10 \mathrm{dBm}$, with a detection limit of $-70 \mathrm{dBm}$, is unable to gather a usable signal from devices buried even only $30 \mathrm{~cm}$ deep. The situation was improved by inserting an electromagnetic waveguide in the hole in the soil by inserting a conducting wire. Notice that no electrical connection is provided between this metallic wire 
and the sensor on one side, or the interrogation unit on the other side, meaning that this setup is resistant to soil motion, oxidation or surface disturbances such a lawn mowing (Fig. 1). The $80 \mathrm{~cm}$ deep sensor was soldered to a RG174 coaxial cable protruding from the hole in the ground as a open feed connexion. All $10 \mathrm{~cm}$ diameter holes were refilled with the same clay of the surrounding area and watered to avoid any air gap.

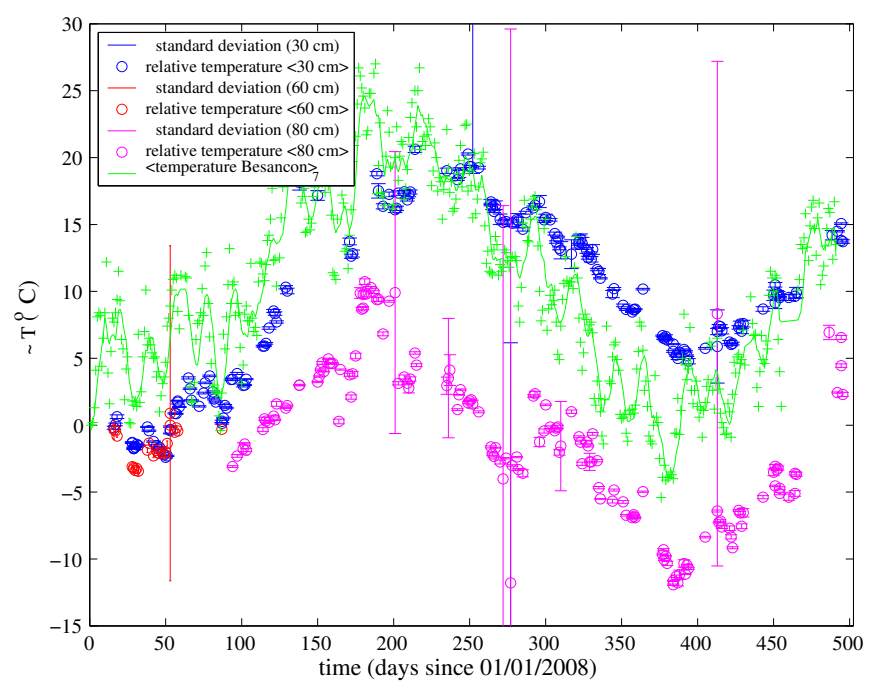

Fig. 2. Evolution over more than one year of the temperature of buried sensors at depths between 30 and $80 \mathrm{~cm}$. The sensors survived this environment for the duration of this experiment, with no noticeable drift or loss in radiofrequency link quality, while providing data consistent with surface temperatures. Only relative temperatures are provided by the sensors since no calibration was performed prior to the experiment.

This setup provided relative temperatures informations (Fig. 2) over time since the sensors had not been calibrated prior to the experiment. The evolution of the temperature provided by the buried sensors is consistent with a sliding average over several weeks of surface temperatures as provided on the web site http: //www. meteo-franche-comte.fr/.

\section{INTERROGATING DELAY LINES}

The simplest implementation of RADAR interrogation units are designed to generate a short - ideally single - pulse including as much energy as possible. This result is achieved by, for example in the radiofrequency range, slowly loading a capacitor with a high voltage (provided by a switching power supply for embedded designs) and "instantaneously" emptying this energy in an antenna through an avalanche transistor. The duration of the energy transfer is defined by the antenna impedance, which is itself designed by the antenna dimensions and surrounding medium permittivity (Fig. 3). Any impedance mismatch between the transistor output and antenna through a balun will induce ringing and, in classical RADAR applications, unwanted additional oscillations beyond the main pulse. This ringing is suitable for interrogating delay lines since more than a single pulse is necessary to efficiently load energy into the acoustic sensor. The extreme case is the resonator of quality factor $Q$ which needs $Q / \pi \simeq 2000$ (at $433 \mathrm{MHz}$ with $Q \simeq 6000$ ) periods to be efficiently loaded: the quality factor of the antenna is usually much bellow this value, of the order of unity, and hence a passive resonator (coaxial line) might be added between the balun and the antenna to store energy and induce enough ringing when interrogating resonators.

As an example of such measurement, a Mala Ramac GPR provides a $100 \mathrm{MHz}$ bistatic configuration which, when used on ice to monitor the bedrock interface of a glacier, allows the identification of a usable signal more than $100 \mathrm{~m}$ deep (Fig. $3)$.

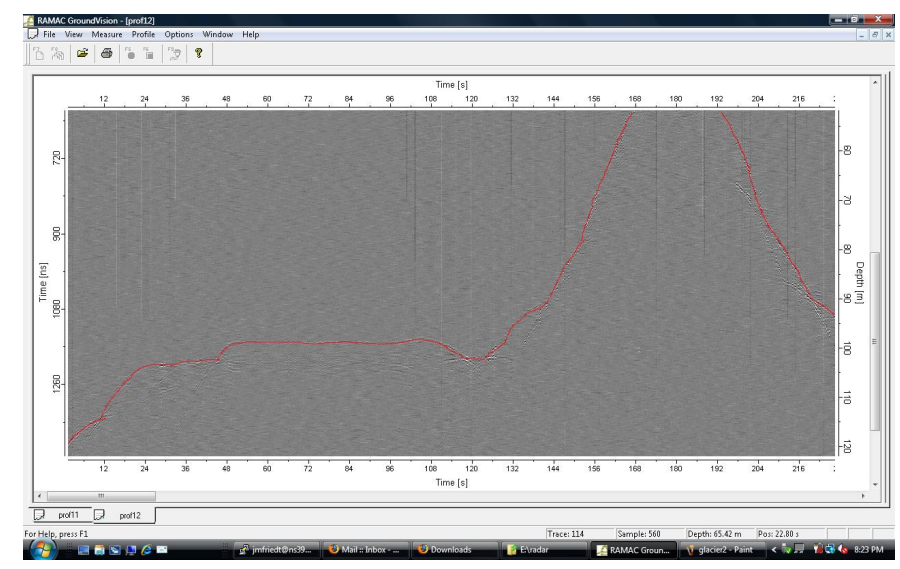

Fig. 3. $100 \mathrm{MHz}$ ground penetrating radar scans of the ice-rock interface: the signal is detected for an interface deeper than $100 \mathrm{~m}$. The red line emphasizing the bedrock interface was added manually for clarity.

Interrogating delay lines using a RADAR setup includes new challenges. We demonstrate experimentally (Fig. 4) that the best performances of a delay line are achieved when the number of periods in the probing signal is equal to the number of finger pairs in the transducer. Below this value, sub-optimal energy transfer occurs and the signal level is too low. Above this value, the echo pulses from the delay line are unnecessarily lengthened and make the identification of time delay more difficult on these extended pulses. Controlling the number of periods on a freely ringing antenna is hardly reproducible since the dielectric environment of the mobile emitting antenna changes during a spatial scan in the field.

We hence focus on a more reproducible and deterministic approach of gating an RF emitter. The hardware we used is the Universal Software Radio Peripheral (USRP, www. ettus.com), and more specifically the RFX900 800 to $1000 \mathrm{MHz} 200 \mathrm{~mW}$ transceiver, with software developed using the opensource tools provided by the GNURadio programs. The acoustic delay line used in this demonstration are those used in Kongsberg's Sentry temperature monitoring system: the center frequency of these transducers in $866 \mathrm{MHz}$, obviously unsuitable for buried sensor purposes but compatible with the hardware available for this demonstration. A single RFX900 daughter board allows for a monostatic interrogation strategy after removing the band pass filter on the emission which is usually installed to comply with American FCC 
regulations.

In order to demonstrate our ability to gather and process reflection signal from a delay line in a monostatic antenna configuration, a wired connection between the transceiver port of the RFX900 daughter board and delay line is provided. We generate $200 \mathrm{~ns}$ pulses (the shortest the available software is able to reliably generate) and monitor the response using the onboard analog to digital converter. Once the I and Q components of the delay line are recorded (16 repetitions of the same interrogation sequence are acquired to provide enough samples for averaging during the post-processing step), magnitude and phase of the returned signals are computed and the time delay between each echo is identified by second-order polynomial fitting of the magnitude envelope. Although Reindl \& al. [3], [4] have demonstrated the greater accuracy achieved by combining phase estimates this particular set of experiment did not provide reliable phase information, probably because of the too low received signal strength which was insufficient to trigger the phase detection of the I/Q demodulator

\section{RANGE ESTIMATE}

Considering the usable reflections recorded from ice-rock interfaces more than $100 \mathrm{~m}$ below the surface (Fig. 3), we wish to estimate the depth [5] at which a GPR-like interrogation scheme would be able to gather informations from a buried delay line. Based on the reflection coefficient of the permittivity mismatch at the interface between the two layers and the insertion loss of delay lines, we can estimate the range at which a delay line will provide the receiver of the radar with enough power for a measurement:

- assuming a plane wave reaching an interface between ice and rock, the Fresnel reflection coefficient $R$ is computed using relative permittivities $\varepsilon_{i c e}=3.1[6]$ and $\varepsilon_{\text {rock }} \simeq 5$ as $R=\left(\frac{\sqrt{\varepsilon_{i c e}}-\sqrt{\varepsilon_{\text {rock }}}}{\sqrt{\varepsilon_{\text {ice }}}+\sqrt{\varepsilon_{\text {rock }}}}\right)^{2}$. We deduce that in this case, the ice-rock interface displays a $-19 \mathrm{~dB}$ reflection coefficient

- the ice-rock interface hence displays a much greater reflection coefficient than the typical delay line with a $S_{11}$ insertion loss at $-35 \mathrm{~dB}$, meaning that the delay line must be closer to the radar to provide a meaningful signal

- the Free Space Propagation Loss (FSPL [7]) calculation $20 \log _{10} d+20 \log _{10} f+32.44$ ( $f$ in $\mathrm{MHz}$ and $d$ in $\mathrm{km}$ ) shows that at $100 \mathrm{MHz}, \operatorname{FSPL}(2 \times 100 \mathrm{~m}) \simeq 58 \mathrm{~dB}$. The $16 \mathrm{~dB}$ difference between the dielectric interface and delay line is compensated for by bringing the reflector at a distance of $15 \mathrm{~m}$ from the radar since $\operatorname{FSPL}(2 \times 15 \mathrm{~m})$ $\simeq 42 \mathrm{~dB}$.

The conclusion of this plane wave analysis is that a SAW delay line buried in ice at a depth of $15 \mathrm{~m}$ should provide the same signal level than the dielectric interface at $100 \mathrm{~m}$. The delay line signature in an echo v.s antenna position graphics (as showin in Fig. 3 for example) is characterized by multiple hyperbola translated in time towards greater depths since the acoustic signal is an attenuated replica of the electromagnetic pulse delayed a few microseconds in time. An intercorrelation

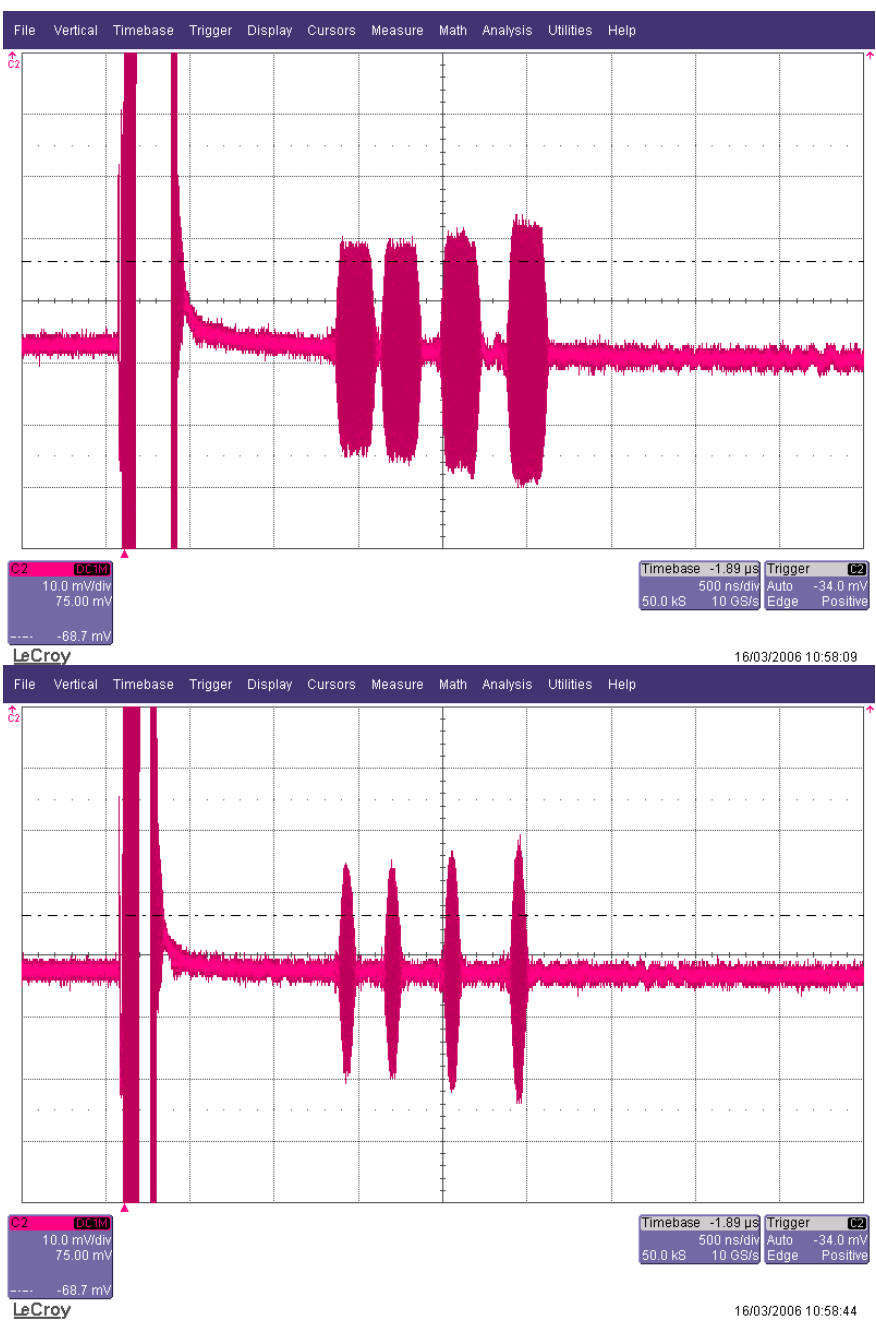

Fig. 4. Two examples of echos observed on the delay line reflection signal, as a function of excitation pulse length. Too long a pulse blurs the echos and makes the identification of the time delay more difficult, without increasing the reflected signal level: this experimental result is consistent with the matched filter theory which states that the optimal number of periods is equal to the number of interdigitated fingers in the transducer. The hardware used in this experiment is unable to generate an excitation pulse with less periods than the number of interdigitated fingers in the transducer of the delay line.

between the various pulses will thus allow accurate identification of the time delays within the delay line and hence identification of the physical quantity affecting these delays.

\section{FDTD SIMULATIONS}

The plane wave approximation is a rough approximation hinting at the depth at which a SAW device can be interrogated, but a more accurate analysis is necessary for an accurate estimate and to later allow a signal identification in which the acoustic contribution is separated from the clutter of the electromagnetic echos. To this end, we have developed two Finite Difference Time Domain analysis, a 2D model with a computation fast enough to allow spatial analysis of the echos as the radar is scanned over dielectric interfaces and a pointlike delay line, and a 3D model for an accurate estimate of 

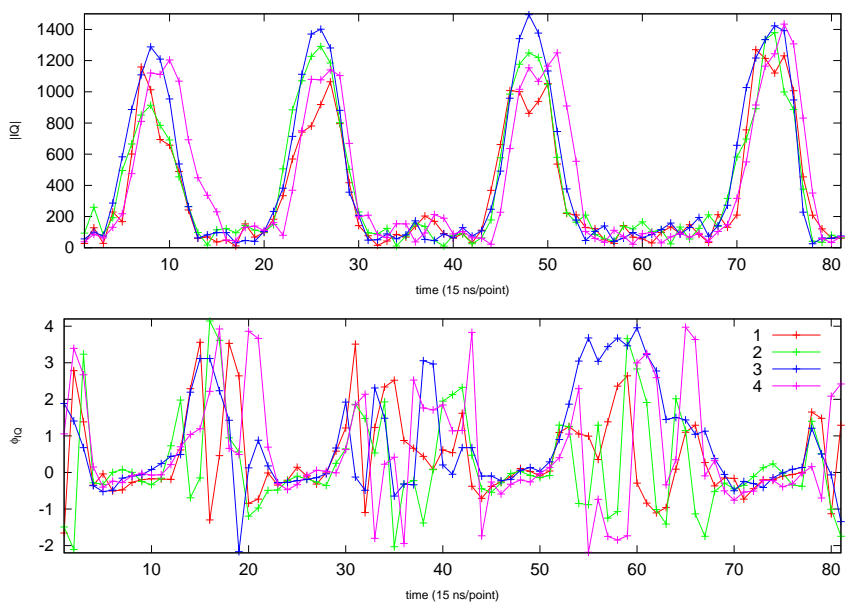

Fig. 5. The digitized I/Q components of the responses of the delay line, sampled at a rate of $64 \mathrm{MHz}$. While the reflection peaks are clearly observed on the magnitude, the received power is insufficient to allow the determination of the phase.
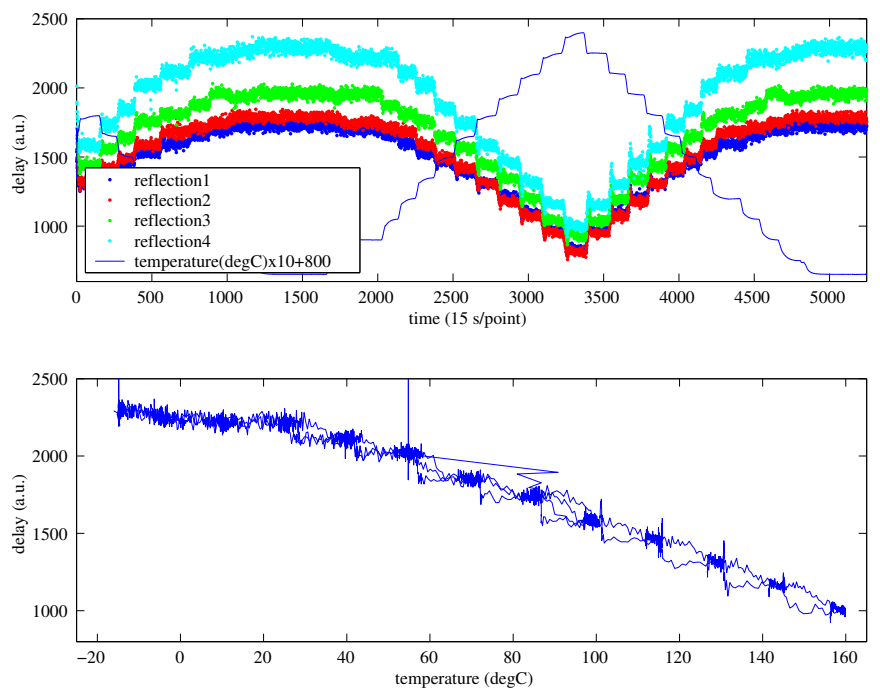

Fig. 6. Evolution of the pulses position as a function of temperature - in the $-20^{\circ} \mathrm{C}$ to $+160^{\circ} \mathrm{C}$ range - based on the received power magnitude position identification after fitting the reflected peaks with second order polynomials and resampling for accurate positioning of the reflection positions.

the propagation losses with accurate distance squared energy distribution.

The FDTD method is a well known numerical tool to compute electromagnetic field propagation in a heterogeneous window [8]. It is based on the Yee scheme [9]. Here, we just present the different used parameters for both the two and three dimensional cases. To achieve the numerical analysis, we must follow the different rules of discretization and stability according to the FDTD method.

Thus, we define spatial and time discretization as mentioned in equation (1). This equation is defined for a $n$-dimensional case. $\lambda$ and $c$ are respectively the smallest wavelength and highest velocity of the electromagnetic field, $\Delta x_{n}$ and $\Delta t$ are respectively the spatial (in each $x_{n}$ directions) and temporal discretization steps.

$$
\Delta x_{n}<\frac{\lambda}{20}, \Delta t<\frac{1}{c \sqrt{\sum_{n=1}^{N} \frac{1}{\left(\Delta x_{n}\right)^{2}}}}
$$

For instance, the Mala Ramac GPR used in the above experience to scan an ice-rock interface provides a wave at $100 \mathrm{MHz}$ in air, i.e., $\lambda_{a}=3 \mathrm{~m}$. However, the rock medium has a higher permittivity than air $\left(\varepsilon_{a}=1\right.$ and $\left.\varepsilon_{s}=5\right)$. So, the smallest wavelength is obtained in rock and is equal to $\lambda_{s} \simeq 1.3 \mathrm{~m}$. By the equation (1), the spatial steps get $\Delta x_{n}=6 \mathrm{~cm}$. Moreover, air provides the highest electromagnetic field velocity $c$. Thus, we choose $\Delta t=\frac{\Delta x_{n}}{2 c \sqrt{3}}$, that is, $\Delta t \simeq 79 \mathrm{ps}$ for $2 \mathrm{D}$ case and $\Delta t \simeq 64 \mathrm{ps}$ for $3 \mathrm{D}$ case. If we change the studied media, all these quantities must be adapted.

The source description is also a critical point of the FDTD method as well as in the time domain as in the spatial one. In time domain, we define the incident electromagnetic field envelop as a Gaussian function. This function is defined by a temporal spread $\omega_{t}$ allowing around ten oscillations for the highest wave length, i.e., $10 \mathrm{~ns}<\omega_{t}<30$ ns. However, to match as well as possible the above experiences, we should defined a temporal spread providing a $200 \mathrm{~ns}$ pulse, that is, $\omega_{t} \simeq 70 \mathrm{~ns}$. Anyway, the second spread being bigger than the basic first one, we can choose either as the one or the other. In spatial Domain, we separately discuss the 2D and 3D cases. First, a radiating dipole stand for the 2D source. This dipole is defined by a hard source in a single cell of the FDTD main grid [8]. Next, a hard source also stands for the source in the 3D case. However, it is not only in a single cell but in a extended area delineated by a 2D Gaussian function. This function is written in the air-rock interface and its spreads stand for the radar half dimensions into the both axes of this plan.

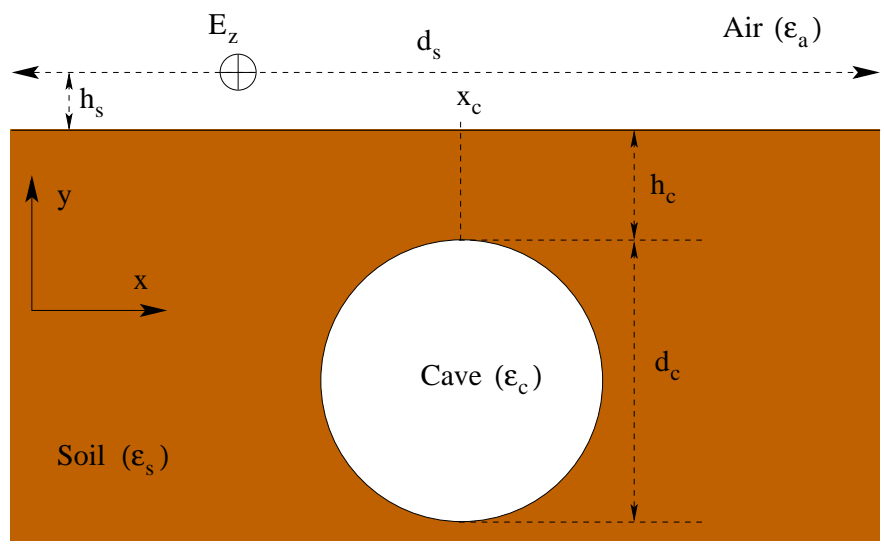

Fig. 7. 2D model used to characterize the interaction between the incident electromagnetic wave and a cave.

We are interested in the interaction between the electromagnetic wave come from the GPR and the soil singularities like caves. We also wish to be able to interpret the GPR experimental results and to predict the losses due to the reflection and diffraction phenomena. Thus, we choose to analyze the 
experiment depicted in figure 7. The GPR is dragged on the ice-rock interface in air $\left(h_{s}=0\right.$ and $\left.d_{s}=14 \mathrm{~m}\right)$. The source is TE polarized and $\lambda=3 \mathrm{~m}$. The air permittivity is equal to unity and the soil one is assumed $\varepsilon_{s}=13$. The incident wave propagates in soil from the GPR to the cave. The distance from the interface to the top of the cave is $h_{c}$. In 2D case, the cave is depicted by an infinite $d_{c}$ diameter cylinder. The cave is filled by air. So, the wave is reflected on the cave surface and goes back to the GPR which is also the detector. By computing the diffracted electromagnetic field at the source position, we are able to simulate the stored signal by the GPR.

In figures 8 to 10 , we show some significant numerical results by plotting the variation of the electromagnetic field square modulus versus both time and source position. We vary two parameters letting the others unchanged. In figure 8 , we put $d_{c}=60 \mathrm{~cm}$ and $h_{c}=10 \mathrm{~m}$. In figure 9, we let the depth of the reflector unchanged and increase the diameter to $d_{c}=2 \mathrm{~m}$. At last, in figure 10, we decrease the depth to $h_{c}=5 \mathrm{~m}$.

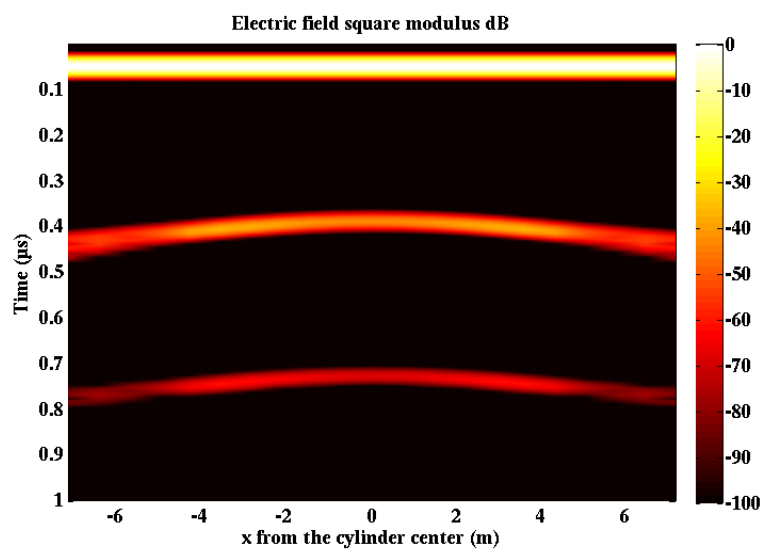

Fig. 8. Variations of the electric field square modulus temporal evolution versus the GPR position from the cave center. A thirty centimeters radius cave is buried ten meters deep and $\lambda_{a}=3 \mathrm{~m}$.

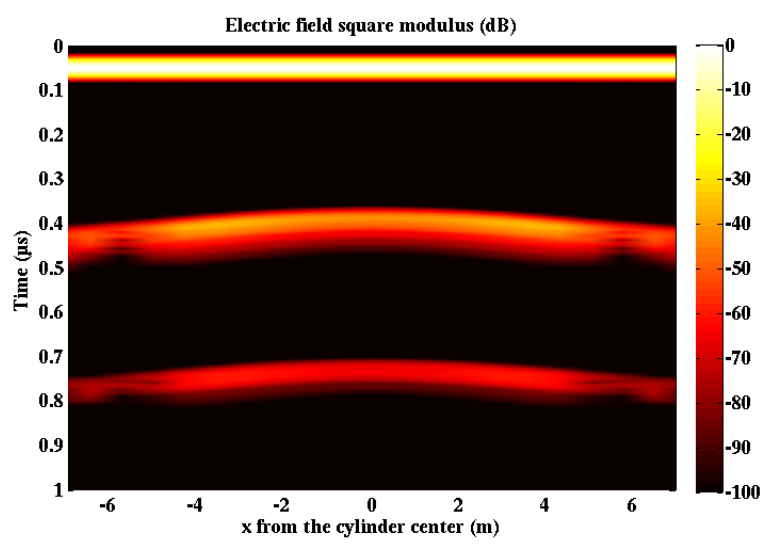

Fig. 9. Variations of the electric field square modulus temporal evolution versus the GPR position from the cave center. A one meter radius cave is buried ten meters deep and $\lambda_{a}=3 \mathrm{~m}$.

First, these results show that the expected experimental

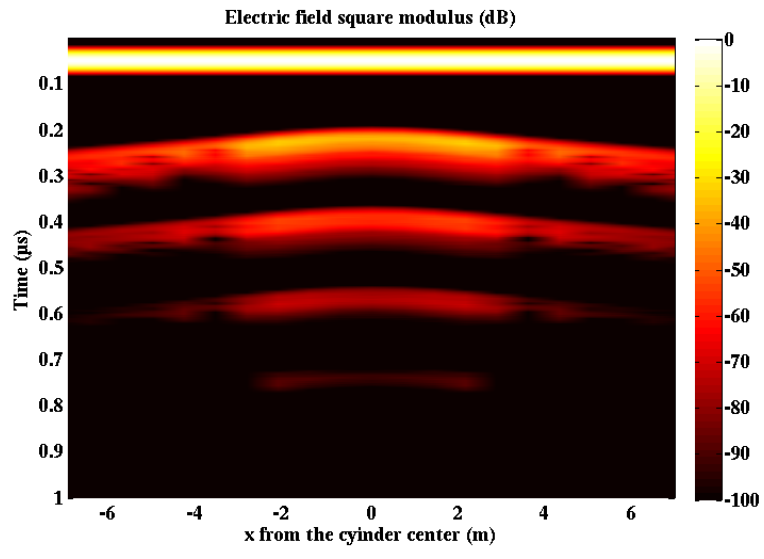

Fig. 10. Variations of the electric field square modulus temporal evolution versus the GPR position from the cave center. A one meter radius cave is buried five meters deep and $\lambda_{a}=3 \mathrm{~m}$.

signal should appear as multiple hyperbola. A brief analysis provides the analytical formula of the source cave distance $d_{s c}$ in equation (2) where $x_{s}$ is the source-sensor position.

$$
d_{s c}=2\left(\sqrt{x_{s}^{2}+\left(h_{c}+\frac{d_{c}}{2}\right)^{2}}-\frac{d_{c}}{2}\right)
$$

This equation is a hyperbola function. This result matches the previous numerical one. A second relevant result is the time or distance between two consecutive hyperbola is equal to the cave depth. Indeed, this distance in figure 8 and 9 is twice over the one in figure 10 and as we can see the time is twice over in the two first figures. These lapses are perfectly equal to the results obtained by the analytical way : $T=2 d_{c} c_{0} \sqrt{\varepsilon_{s}}\left(c_{0}\right.$ is the vacuum light velocity). From these results, we could have also discussed the losses due to the reflection and the diffraction into the soil. However, we use a cross section of the previous results. In figure 11 , we plot the temporal evolution of the electric field square modulus when the source is placed just above the cave. These results are obtained for (a) an infinite radius, 5 meters deep, (b) $30 \mathrm{~cm}$ radius, 5 meters deep, (c) $30 \mathrm{~cm}$ radius, 10 meters deep. It is obvious the losses are strongly dependent on the cave size but also on the cave depth (even if it is a little bit less sensible). Indeed, the losses in the (b) case are $15 \mathrm{~dB}$ over those of the (a) one, i.e., more the cave size decreases and more the losses increase. At last, we also show in figure 11 a $3 \mathrm{D}$ result the (d) case. Thus we confirm the previous result on the correlation between the cave size and the losses. In fact, the cave is now depicted by a $30 \mathrm{~cm}$ radius sphere and thus the efficient surface become smaller. The losses are $10 \mathrm{~dB}$ more important in this new case than in the previous (b) one. As a last remark, we can say that the difference between the source width in $3 \mathrm{D}$ and $2 \mathrm{D}$ cases is due to the choice of different value for the spread of the incident pulse. This is not a problem for the discussion about the losses

In further works, we will discuss more specially about the $3 \mathrm{D}$ cases and we also introduce the losses due to the 


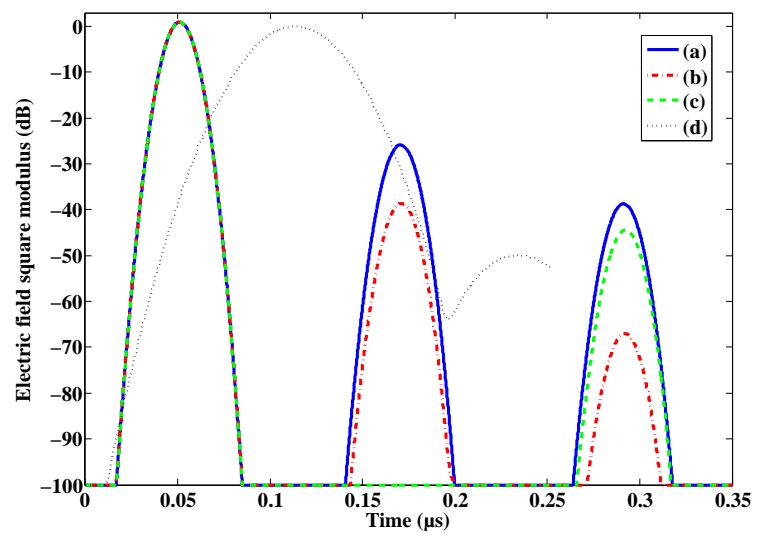

Fig. 11. The electric field square modulus temporal evolution for several cave radii and several depths. (a) infinite radius, 5 meters deep. (b) $30 \mathrm{~cm}$ radius, 5 meters deep. (c) $30 \mathrm{~cm}$ radius, 10 meters deep. (a), (b), (c) are 2D numerical analysis with $\omega_{t}=10 \mathrm{~ns}$. (d) $30 \mathrm{~cm}$ radius, 5 meters deep in a 3D numerical analysis with $\omega_{t}=30 \mathrm{~ns}$

absorption by using already implemented model for dispersive and absorbing media in [10], [11]. However, we are already able to understand and predict the experimental results of GPR experiments.

\section{CONCLUSION}

We have demonstrated that SAW resonators packaged in ceramic packages withstand the environment if sensors buried in clay for over a year. Regular monitoring of these buried devices provided temperature evolutions consistent with surface temperatures, although the interrogation unit compliant with the $434 \mathrm{MHz}$ European ISM band did not allow the sensors to be buried more than $80 \mathrm{~cm}$ deep.

In order to improve the interrogation depth of sensors, we have analyzed the interrogation strategy of ground penetrating radars, able to gather informations of reflected electromagnetic energy at dielectric interfaces up to $100 \mathrm{~m}$ deep at $100 \mathrm{MHz}$ in low loss propagation media such as ice. We extend this result to an estimate of the depth at which a SAW delay line might provide the same amount of reflected energy by compensating the larger insertion loss by brining the sensor closer to the surface: a plane wave calculation of Fresnel reflection coefficient hints at a possible depth of $15 \mathrm{~m}$.

Further perspectives of this work include switching from a monostatic delay line interrogation configuration to a bistatic configuration as allowed by installing a second RFX900 daughter board on the USRP, as well as attempting a bistatic resonator interrogation configuration which allows generating much larger output power than the current $10 \mathrm{dBm}$.

\section{ACKNOWLEDGMENT}

J.-MF would like to thank S. Zhgoon (Moscow Power Engineering Institute, Russia) for fruitful discussions during the 2009 IFCS/EFTF conference. Ground Penetrating Radar measurements on glaciers was performed as part of the HydroSensor-FLOWS program under the supervision of D. Laffly, C. Marlin, M. Griselin.

\section{REFERENCES}

[1] S. Gogineni, D. Tammana, D. Braaten, C. Leuschen, T. Akins, J. Legarsky, P. Kanagaratnam, J. Stiles, C. Allen, , and K. Jezek, "Coherent radar ice thickness measurements over the greenland ice sheet," J. of Geophysical Research, vol. 106, no. D24, p. 3376133772, 2001.

[2] C. Allen, K. Shi, and R. Plumb, "Characterization of a cooperative target for ground-penetrating radar," IEEE Transactions on Geoscience and Remote Sensing, vol. 36, no. 5, pp. 1821-1825, 1998.

[3] L. Reindl and I. Shrena, "Wireless measurement of temperature using surface acoustic waves sensors," IEEE transactions on ultrasonics, ferroelectrics, and frequency control, vol. 51, no. 11, november 2004.

[4] S. Schuster, S. Scheiblhofer, L. Reindl, and A. Stelzer, "Performance evaluation of algorithms for SAW-based temperature measurement," IEEE transactions on ultrasonics, ferroelectrics, and frequency control, vol. 53, no. 6, june 2006.

[5] G. Leucci, "Ground penetrating radar: the electromagnetic signal attenuation and maximum penetration depth," Scholarly research exchange, no. 6, 2008.

[6] J. Jiang and D. Wu, "Ice and water permittivities for millimeter and submillimeter remote sensing applications," Atmospheric Science Letters, vol. 5, pp. 146-151, 2004.

[7] C. Balanis, Antenna theory - analysis and design, 2nd Ed. John Wiley \& Sons, 1997.

[8] A. Taflove and S. Hagness, Computational electrodynamics: The finite difference time domain method, 2nd ed. Northwood, London: Artech House Publishers, 2000.

[9] K. Yee, "Numerical solution of initial boundary value problems involving Maxwell's equations," IEEE Trans. Ant. Prop., vol. 14, pp. 302-7, 1966.

[10] T. Laroche and A. Vial, "FDTD spectroscopic study of metallic nanostructures: on the pertinent employment of tabulated permittivities," in One-Dimensional Nanostructures, Z. Wang, Ed. Springer, 2008, pp. $147-173$.

[11] A. Vial and T. Laroche, "Description of dispersion properties of metals by mean of the critical points model and application to the study of resonant structures using the FDTD method," J. Phys. D: Appl. Phys., vol. 40, pp. 7152-7158, 2007. 